[RAdiocarbon, Vol 26, No. 3, 1984, P 384-391]

\title{
INSTITUT ROYAL DU PATRIMOINE ARTISTIQUE RADIOCARBON DATES $\mathbf{X}$
}

\section{MICHELLE DAUCHO'T-DEHON, MARK VAN STRYDONCK, and JOS HEYLEN}

Institut royal du Patrimoine artistique, Koninklijk Instituut voor het

Kunstpatrimonium, Brussels, Belgium

This list contains the results of ${ }^{14} \mathrm{C}$ determinations obtained at the laboratory in 1982-1983.

\section{ACKNOWLEDGMENTS}

The authors would like to thank the ${ }^{14} \mathrm{C}$ laboratory in Lyon for the $\delta^{13} \mathrm{C}$ measurement. M Dupas is gratefully acknowledged for analysis of mortar samples.

\section{Doel-Beveren series}

\section{GEOLOGIC SAMPLES}

\section{Belgium}

Peat and wood from Doel-Beveren in $\mathrm{O}$ Vlaanderen $\left(51^{\circ} 18^{\prime} \mathrm{N}, 4^{\circ}\right.$ 15' E). Coll Sept 1981 and subm Oct 1982 by C Verbruggen, Univ Gent, Belgium.

IRPA-454. III

$4900 \pm 60$

Peat from base of thin clay layer at $350 \mathrm{~cm}$ depth. Comment (CV): date is probably correct.

IRPA-455. I

$2050 \pm 70$

Peat from top of peat layer at $200 \mathrm{~cm}$ depth. Comment $(\mathrm{CV})$ : date agrees with extension of Fagus in pollen diagram, typical for that period.

IRPA-456. I

$3000 \pm 70$

Wood from top of peat layer at $200 \mathrm{~cm}$ depth. Comment (CV): date similar to IRPA-455 was expected.

IRPA-457. II

$\mathbf{5 3 5 0} \pm \mathbf{7 0}$

Peat from top of thin layer at $338 \mathrm{~cm}$ depth. Comment (CV): date is too old compared to IRPA-454 at base of layer.

IRPA-458. IV

Peat from base of peat layer at $412 \mathrm{~cm}$ depth. Comment (CV): date confirms onset of peat growth in lower Scheldt-basin.

\section{Assenede series}

Peat from Holocene sediment in de Schelde polders at Assenede in O Vlaanderen $\left(51^{\circ} 16^{\prime} \mathrm{N}, 3^{\circ} 47^{\prime} \mathrm{E}\right)$. Coll and subm Sept 1982 by $\mathrm{C}$ Baeteman, Geol Service, Belgium.

IRPA-487. B1

$3130 \pm 60$

Peat from 168 to $183 \mathrm{~cm}$. 
IRPA-488. (1) B87

$3790 \pm 60$

Peat from 155 to $180 \mathrm{~cm}$. Distillation product.

IRPA-488. (2) $\mathrm{B87}$

$3900 \pm 60$

Peat from 155 to $180 \mathrm{~cm}$.

IRPA-489. B5

$3090 \pm 60$

Peat from 140 to $150 \mathrm{~cm}$.

IRPA-567. DB3

$4560 \pm 70$

Peat from 155 to $160 \mathrm{~cm}$.

IRPA-568. DB4

$2230 \pm 50$

Peat from 185 to $190 \mathrm{~cm}$.

General Comment (CB): IRPA-567 is much older than other dates, most probably due to boring (DB3) in strong micro-relief of top of cover sand. IRPA-568 is much younger than expected most probably due to contamination by younger roots.

\section{Western coastal plain of Belgium series}

Peat and wood from several levels of core in W Vlaanderen. Dated to study evolution of so-called surface peat (Baeteman et al, 1979; Baeteman, ms). Coll and subm 1982 by C Baeteman.

IRPA-524. Driegrachten 1-A

$3610 \pm 60$

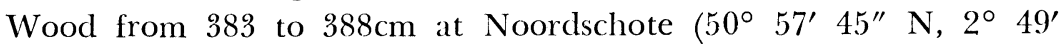
$\left.22^{\prime \prime} \mathrm{E}\right)$.

IRPA-529. Driegrachten 1-B

$3540 \pm 60$

Peat from 383 to $388 \mathrm{~cm}$ at Noordschote $\left(50^{\circ} 57^{\prime} 45^{\prime \prime} \mathrm{N}, 2^{\circ} 49^{\prime} 22^{\prime \prime} \mathrm{E}\right)$. General Comment (LD): samples date slight increase of marine influence corresponding with end of peat formation in some parts of coastal plain.

IRPA-530. Driegrachten 2

$3790 \pm 60$

Peat from 463 to $467 \mathrm{~cm}$ at Noordschote ( $\left.50^{\circ} 57^{\prime} 45^{\prime \prime} \mathrm{N}, 2^{\circ} 49^{\prime} 22^{\prime \prime} \mathrm{E}\right)$. Comment (LD): dates possible dry period indicated by diatom analysis.

IRPA-531. Driegrachten 3

$5220 \pm 70$

Peat from 611 to $617 \mathrm{~cm}$ at Noordschote $\left(50^{\circ} 57^{\prime} 45^{\prime \prime} \mathrm{N}, 2^{\circ} 49^{\prime} 22^{\prime \prime} \mathrm{E}\right)$.

IRPA-532. Pervijse Orthodoxe kerk 1

$5130 \pm 70$

Peat from 408 to $411 \mathrm{~cm}$ at Lampernisse $\left(51^{\circ} 03^{\prime} 20^{\prime \prime} \mathrm{N}, 2^{\circ} 47^{\prime} 32^{\prime \prime} \mathrm{E}\right)$.

IRPA-538. Jacobs 1

$\mathbf{5 3 6 0} \pm \mathbf{7 0}$

Peat from 410 to $412 \mathrm{~cm}$ at Oudekapelle $\left(51^{\circ} 01^{\prime} 11^{\prime \prime} \mathrm{N}, 2^{\circ} 48^{\prime} 10^{\prime \prime} \mathrm{E}\right)$.

General Comment (CB): samples date onset of freshwater predominance and early start of continuous peat growth in region. Dates are as expected. They coincide well and show that peat growth started slightly earlier in land areas than in surrounding areas. 
IRPA-537. Jacobs 2

$1870 \pm 60$

Peat from 217 to $220 \mathrm{~cm}$ at Oudekapelle $\left(51^{\circ} 01^{\prime} 11^{\prime \prime} \mathrm{N}, 2^{\circ} 48^{\prime} 10^{\prime \prime} \mathrm{E}\right)$. Comment (CB): age indicates that end of peat growth in area is later than in surrounding areas. Date agrees with expected ages as other ${ }^{14} \mathrm{C}$ dates indicated that in this area peat could grow much longer than in surrounding coastal plain.

IRPA-533. Pervijse Orthodoxe kerk $2 \quad \mathbf{7 2 3 0} \pm \mathbf{7 0}$ Peat from 882 to $885 \mathrm{~cm}$ at Lampernisse ( $\left.51^{\circ} 03^{\prime} 20^{\prime \prime} \mathrm{N}, 2^{\circ} 47^{\prime} 32^{\prime \prime} \mathrm{E}\right)$.

IRPA-534. Pervijse Orthodoxe kerk 3

$7110 \pm 90$

Peat from 875 to $879 \mathrm{~cm}$ at Lampernisse $\left(51^{\circ} 03^{\prime} 20^{\prime \prime} \mathrm{N}, 2^{\circ} 47^{\prime} 32^{\prime \prime} \mathrm{E}\right)$.

IRPA-541. Dijk 1

$6680 \pm 80$

Peat from 816 to $819 \mathrm{~cm}$ at Oudekapelle $\left(51^{\circ} 01^{\prime} 11^{\prime \prime} \mathrm{N}, 2^{\circ} 48^{\prime} 10^{\prime \prime} \mathrm{E}\right)$.

IRPA-542. Dijk 2

$6870 \pm 80$

Peat from 833 to $836 \mathrm{~cm}$ at Oudekapelle $\left(51^{\circ} 01^{\prime} 11^{\prime \prime} \mathrm{N}, 2^{\circ} 48^{\prime} 10^{\prime \prime} \mathrm{E}\right)$. General Comment (CB): top and base of basal peat showed that initial marine influence in area was already occurring at beginning of Atlantic period. Dates agree with expected ages in relation to depth.

\section{Oostkerke series}

Peat from Oostkerke, W Vlaanderen ( $\left.51^{\circ} 02^{\prime} 40^{\prime \prime} \mathrm{N}, 2^{\circ} 47^{\prime} 30^{\prime \prime} \mathrm{E}\right)$. Coll and subm Sept 1982 by C Baeteman.

$\begin{array}{lll}\text { IRPA-535. } & 12.24 \text { to } 12.27 \mathrm{~m} & 6750 \pm 80 \\ \text { IRPA-536. } & 12.34 \text { to } 12.38 \mathrm{~m} & \mathbf{7 0 0 0} \pm 80\end{array}$

General Comment $(\mathrm{CB})$ : samples date top and base of deepest intercalated peat layer, as yet known, in coastal plain in first part of Atlantic period.

\section{Avekapelle series}

Peat from Avekapelle, W Vlaanderen ( $\left.51^{\circ} 03^{\prime} 55^{\prime \prime} \mathrm{N}, 2^{\circ} 45^{\prime} 55^{\prime \prime} \mathrm{E}\right)$. Coll and subm Sept 1982 by C Baeteman.

\section{IRPA-539. 983 to $990 \mathrm{~cm}$$$
3890 \pm 70
$$ \\ IRPA-540. 760 to $768 \mathrm{~cm}$ \\ $2680 \pm 60$}

General Comment (CB): both samples were coll from same tidal channel sequence at rather great depth $(-5.7 \mathrm{~m}$ and $-3.5 \mathrm{~m})$. Ages show that peat is eroding from surface of upper regional peat layer and not from second regional peat layer occurring at greater depth $(-2.5 \mathrm{~m})$.

\section{Wulpen series}

Peat from Wulpen in W Vlaanderen $\left(51^{\circ} 06^{\prime} \mathrm{N}, 2^{\circ} 42^{\prime} 45^{\prime \prime} \mathrm{E}\right)$. Coll and subm March 1982 by $\mathrm{C}$ Baeteman. Results used to study stratigraphy in Western coastal plain.

IRPA-527. Wulpen A-1

$$
3490 \pm 60
$$

Base of upper peat layer at $117 \mathrm{~cm}$ below surface. 
IRPA-528. Wulpen A-2

$2970 \pm 60$

Top of upper peat layer at $100 \mathrm{~cm}$ below surface.

General Comment (CB): samples coll from top and base of upper part of "surface peat" in area divided by intercalated clay layer, corresponding with CIV-B transgression. Age of top coincides well with all other data indicating end of surface peat in $\mathrm{W}$ coastal plain. Age of base coincides with some dates of similar series in same area (Baeteman et al, 1979).

\section{IRPA-512. Raversyde}

$2580 \pm 60$

Peat from top layer at $349 \mathrm{~cm}$ below surface in W Vlaanderen $\left(51^{\circ}\right.$ $11^{\prime} 45^{\prime \prime} \mathrm{N}, 2^{\prime} 52^{\prime} 20^{\prime \prime} \mathrm{E}$ ). Coll and subm Jan 1983 by C Baeteman.

\section{Kallo series}

Peat and wood from Kallo, Antwerpen (51 $\left.11^{\circ} 45^{\prime \prime} \mathrm{N}, 4^{\circ} 14^{\prime} 31^{\prime \prime} \mathrm{E}\right)$. Coll and subm May 1982 by D Ferguson, Univ Antwerpen, Belgium.

IRPA-547. Profile 1

$2530 \pm 70$

Wood at $190 \mathrm{~cm}$ below surface.

IRPA-544. Profile 2-1

$2810 \pm 60$

Top of peat layer at $130 \mathrm{~cm}$ below surface.

IRPA-545. Profile 2-2

$4240 \pm 70$

Middle of peat layer at $227 \mathrm{~cm}$ below surface.

IRPA-546. Profile 2-3

$6790 \pm 80$

Base of peat layer at $347 \mathrm{~cm}$ below surface.

\section{Mark series}

These results complete pub list $(\mathrm{R}, 1981, \mathrm{v} 23, \mathrm{p}$ 345-346; R, 1983, $\mathrm{v} 25$, p 868-869) of samples from alluvial plain of Mark $\mathrm{R}$ in W Vlaanderen and Brabant. Coll and subm 1983 by W Huybrechts, Geol Inst, Free Univ Brussels.

IRPA-506. Galmaarden B80/6/36 Top

$2580 \pm 60$

Clayey peat 229 to $234 \mathrm{~cm}$ below surface $\left(50^{\circ} 45^{\prime} \mathrm{N}, 3^{\circ} 57^{\prime} \mathrm{E}\right)$.

IRPA-548. Galmaarden B80/6/36 Base

$3260 \pm 60$

Clayey peat 280 to $290 \mathrm{~cm}$ below surface $\left(50^{\circ} 45^{\prime} \mathrm{N}, 3^{\circ} 57^{\prime} \mathrm{E}\right)$.

IRPA-549. Galmaarden B82/6/16 Top

$4330 \pm 70$

Clayey peat 362 to $367 \mathrm{~cm}$ below surface $\left(50^{\circ} 45^{\prime} \mathrm{N}, 3^{\circ} 57^{\prime} \mathrm{E}\right)$.

IRPA-550. Galmaarden B81/6/16 Top

$5730 \pm 80$

Clayey peat 320 to $326 \mathrm{~cm}$ below surface $\left(50^{\circ} 45^{\prime} \mathrm{N}, 3^{\circ} 57^{\prime} \mathrm{E}\right)$.

IRPA-551. Galmaarden B81/6/16 Base

$5770 \pm 80$

Clayey peat 341.5 to $350 \mathrm{~cm}$ below surface $\left(50^{\circ} 45^{\prime} \mathrm{N}, 3^{\circ} 57^{\prime} \mathrm{E}\right)$.

IRPA-552. Herne B81/6/5 Middle

$8890 \pm 100$

Wood 622 to $625 \mathrm{~cm}$ below surface $\left(50^{\circ} 43^{\prime} \mathrm{N}, 4^{\circ} 01^{\prime} \mathrm{E}\right)$. 
388 Michèle Dauchot-Dehon, Mark Van Strydonck, and Jos Heylen

IRPA-554. Herne B81/6/5 Base

$10,060 \pm 110$

Residues of wood 695 to $700 \mathrm{~cm}$ below surface $\left(50^{\circ} 43^{\prime} \mathrm{N}, 4^{\circ} 01^{\prime} \mathrm{E}\right)$.

IRPA-556. Herne B81/6/9 Base

$2390 \pm 60$

Wood 195 to $200 \mathrm{~cm}$ below surface $\left(50^{\circ} 43^{\prime} \mathrm{N}, 4^{\circ} 01^{\prime} 15^{\prime \prime} \mathrm{E}\right)$.

IRPA-543. Hulst

Other countries

Wood (trunk) from upper peat layer in Schelde Channel at Hulst, Zeeuws Vlaanderen, Netherlands $\left(51^{\circ} 22^{\prime} \mathrm{N}, 4^{\circ} 13^{\prime} \mathrm{E}\right)$. Coll and subm March 1983 by S Dievoet.

\section{Djelfa series}

Calcareous crusts and organic material from Djelfa, Algeria. Coll and subm Feb 1981 by H Tsaki, Univ Oran, Algeria.

IRPA-451. DJI/E1DZ

$7970 \pm 370$

Organic material from black soil horizon $\left(34^{\circ} 41^{\prime} \mathrm{N}, 3^{\circ} 15^{\prime} \mathrm{E}\right)$ at $850 \mathrm{~cm}$ in profile. Diluted: $26 \%$ sample. Expected age: 7000 to $10,000 \mathrm{BP}$.

IRPA-459. C107/1DCDZ

$21,000 \pm 350$

Calcareous crust from $20 \mathrm{~cm}$ depth $\left(34^{\circ} 52^{\prime} \mathrm{N}, 3^{\circ} 27^{\prime} \mathrm{E}\right)$. Expected age: $>20,000$ BP.

IRPA-460. C88DCDZ

$10,570 \pm 120$

Crusty calcareous tufa from $40 \mathrm{~cm}$ depth $\left(34^{\circ} 50^{\prime} \mathrm{N}, 3^{\circ} 27^{\prime} \mathrm{E}\right)$. Expected age: 10,000 to $15,000 \mathrm{BP}$.

IRPA-461. Cl3bis 1DCDZ

Calcareous crust from $10 \mathrm{~cm}$ depth $\left(34^{\circ} 53^{\prime} \mathrm{N}, 3^{\circ} 26^{\prime} \mathrm{E}\right)$. Expected age: $>30,000$ вр.

IRPA-462. C13/2DCDZ $\quad 26,000 \pm 800$

Crusty calcareous tufa from $25 \mathrm{~cm}$ depth $\left(34^{\circ} 53^{\prime} \mathrm{N}, 3^{\circ} 27^{\prime} \mathrm{E}\right)$. Expected age: $>30,000$ вР.

IRPA-463. C13/1DCDZ

$14,910 \pm 180$

Calcareous crust from $10 \mathrm{~cm}$ depth $\left(34^{\circ} 53^{\prime} \mathrm{N}, 3^{\circ} 27^{\prime} \mathrm{E}\right)$. Expected age: $>25,000$ вр.

IRPA-464. DCI/ODZ

$31,200 \pm 1300$

Calcareous and friable crust from $80 \mathrm{~cm}$ depth $\left(34^{\circ} 53^{\prime} \mathrm{N}, 3^{\circ} 26^{\prime} \mathrm{E}\right)$. Expected age: $>30,000$ вр.

IRPA-465. DCI/00DZ

$31,600 \pm 1300$

Calcareous crust from $30 \mathrm{~cm}$ depth $\left(34^{\circ} 53^{\prime} \mathrm{N}, 3^{\circ} 26^{\prime} \mathrm{E}\right)$. Expected age: $>30,000$ вр.

IRPA-466. DCI/000DZ

$22,200 \pm 430$

Calcareous crust from $10 \mathrm{~cm}$ depth $\left(34^{\circ} 53^{\prime} \mathrm{N}, 3^{\circ} 26^{\prime} \mathrm{E}\right)$. Expected age: $>30,000$ вP. 
Calcareous crust from $30 \mathrm{~cm}$ depth $\left(34^{\circ} 51^{\prime} \mathrm{N}, 3^{\circ} 27^{\prime} \mathrm{E}\right)$. Expected age: $>20,000$ вр.

\section{IRPA-468. DJII/2DZ}

Calcareous crust from $20 \mathrm{~cm}$ depth $\left(34^{\circ} 50^{\prime} \mathrm{N}, 3^{\circ} 20^{\prime} \mathrm{E}\right)$. Expected age: $>40,000$ вр.

\section{IRPA-469. DCI/3DZ}

Calcareous and friable crust from $50 \mathrm{~cm}$ depth $\left(34^{\circ} 51^{\prime} \mathrm{N}, 3^{\circ} 27^{\prime} \mathrm{E}\right)$.

IRPA-470. DJII/7DZ

Calcareous crust, very powdery, from $180 \mathrm{~cm}$ depth $\left(34^{\circ} 40^{\prime} \mathrm{N}, 3^{\circ}\right.$ $20^{\prime}$ E). Expected age: $>40,000 \mathrm{BP}$.

\section{IRPA-471. DJII/4DZ}

$36,800 \pm 2800$

Crusty calcareous tufa from $60 \mathrm{~cm}$ depth $\left(34^{\circ} 40^{\prime} \mathrm{N}, 3^{\circ} 20^{\prime} \mathrm{E}\right)$. Expected age: $>40,000$ вр.

General Comment (HT): all samples are of Würmian or Soltanien age in stratigraphy of North Africa, which indicate that crusts have been redeposited. This can be explained by running water during last period of rain. Chronology of layers follows: crusts (IRPA-460 and -469) and crusts (IRPA-459) of Tensifto-Amirien glacis from middle Quaternary are younger than crust (IRPA-462) and crust (IRPA-466) of Soleto-Moulouyenne outliers from lower Quaternary. Results confirm geomorphologic layers.

\section{IRPA-526. Evergem}

\section{ARCHAEOLOGIC SAMPLES}

\section{Belgium}

Charcoal from Evergem, O Vlaanderen $\left(51^{\circ} 6^{\prime} 36^{\prime \prime} \mathrm{N}, 3^{\circ} 42^{\prime} 32^{\prime \prime} \mathrm{E}\right)$. Coll and subm Dec 1982 by G Verbruggen. Archaeol date: Bronze age.

\section{Destelbergen series}

Charcoal from graves, Destelbergen, $\mathrm{O}$ Vlaanderen $\left(51^{\circ} 03^{\prime} 16^{\prime \prime} \mathrm{N}\right.$, $\left.3^{\circ} 46^{\prime} 40^{\prime \prime} \mathrm{E}\right)$. Coll and subm June 1982 by $\mathrm{H}$ Thoen, Univ Gent, Belgium.

IRPA-476. Grave 84.

$$
\mathbf{2 4 3 0} \pm \mathbf{5 0}
$$

Sample from 64 to $94 \mathrm{~cm}$ depth. Expected age: Bronze or Early Iron age.

IRPA-477. Grave 87

Sample from 67 to $77 \mathrm{~cm}$ depth. Expected age: late Bronze or Early Iron age.

\section{IRPA-505. Webbekom}

Charcoal from Webbekom, Brabant $\left(50^{\circ} 57^{\prime} 54^{\prime \prime} \mathrm{N}, 5^{\circ} 04^{\prime} 26^{\prime \prime} \mathrm{E}\right)$. Coll and subm March 1982 by L Van Impe, Natl Service Excavations, Belgium. Comment: no NaOH pretreatment. Archaeol date: 2650 to 2300 BP. 


\section{Donk series}

Samples from Donk, Limburg $\left(50^{\circ} 56^{\prime} \mathrm{N}, 5^{\circ} 07^{\prime} 30^{\prime \prime} \mathrm{E}\right)$. Coll and subm May 1983 by L Van Impe.

\section{IRPA-507. 82DO554 \\ $1010 \pm 50$}

Charcoal from 80 to $100 \mathrm{~cm}$ depth. Expected date: 2400 to $2200 \mathrm{BP}$.

IRPA-508. 81D0507

$1220 \pm 70$

Charcoal from 90 to $110 \mathrm{~cm}$ depth. Diluted: $41 \%$ sample. Expected date: 3950 to 3450 вр.

IRPA-509. 80DO429

$1740 \pm 80$

Charcoal from 70 to $80 \mathrm{~cm}$ depth. Diluted: $57 \%$ sample. Expected date: 1700 to 1550 вр.

\section{IRPA-510. 81DO515}

$1440 \pm 50$

Wood from well at 185 to $205 \mathrm{~cm}$ below water-bearing bed. Expected date: 2650 to $1550 \mathrm{BP}$.

\section{IRPA-511. 81DO505}

$3110 \pm 60$

Charcoal from 130 to $140 \mathrm{~cm}$ depth. Expected date: 2650 to $2350 \mathrm{BP}$.

\section{IRPA-503. 81 Wellin B2}

$1420 \pm 70$

Charcoal from Wellin, Luxembourg $\left(50^{\circ} 5^{\prime} 2^{\prime \prime} \mathrm{N}, 5^{\circ} 6^{\prime} 54^{\prime \prime} \mathrm{E}\right)$, at $185 \mathrm{~cm}$ below surface. Coll and subm Aug 1981 by A Matthys, Natl Service Excavations. Expected date: 1850 вр.

\section{Ucimont series}

Charcoal from Ucimont, Luxembourg $\left(49^{\circ} 49^{\prime} 54^{\prime \prime} \mathrm{N}, 5^{\circ} 3^{\prime} 21^{\prime \prime} \mathrm{E}\right)$. Coll and subm 1982 by A Matthys. No archaeol data.

IRPA-522. $\quad \mathrm{UC79} / \mathrm{n}^{\circ} 2$

IRPA-523. $\mathrm{UC79} / \mathbf{n}^{\circ} 1$

$$
1390 \pm 50
$$

Modern

Modern

IRPA-525. $77 \mathrm{Cu} 10$

Charcoal from Cugnon, Luxembourg $\left(49^{\circ} 48^{\prime} 9^{\prime \prime} \mathrm{N}, 5^{\circ} 12^{\prime} 14^{\prime \prime} \mathrm{E}\right)$, at $125 \mathrm{~cm}$ below surface. Coll 1977 and subm 1982 by A Matthys.

\section{IRPA-606. Karbonkelhuis}

$610 \pm 50$

Timber from Karbonkelhuis, Antwerpen $\left(51^{\circ} 13^{\prime} 16^{\prime \prime} \mathrm{N}, 4^{\circ} 23^{\prime} 60^{\prime \prime}\right.$ E). Subm Jan 1984 by $\mathrm{P}$ de Henau, Inst Royal Patrimoine artistique. Comment: dated to establish original building 16th century or 19th century rebuilding. Calibrated date (Klein et al, 1982): AD 1270-1410. Since sample was taken from beam, annual rings are missing; accounting also for drying time of wood, date agrees with 16th century construction.

\section{Mortar series}

Dating of mortars has been studied in our lab since 1980. With collaboration of Centre de Datation et d'Analyses isotopiques, Univ Claude Bernard, Lyon, France, we have followed a method based on those of 
Folk and Valastro (1976). Results come from activity measurement of "hypothetical fraction" where no "dead carbonate" has reacted. Isotopic fractionation correction was made (Van Strydonck, Dupas, \& DauchotDehon, in press).

\section{IRPA-296. St Lambert}

Mortar from St Lambert cathedral, Liège $\left(50^{\circ} 38^{\prime} 45^{\prime \prime} \mathrm{N}, 5^{\circ} 34^{\prime} 30^{\prime \prime}\right.$ E). Coll and subm 1979 by H Danthine, Univ Liège. Sample was taken from underground remains of wall constructed between 7 th and 13th century, at $1.8 \mathrm{~m}$ below street level.

IRPA-490. Antwerpen

Mortar from "Onze-Lieve-Vrouw" cathedral, Antwerpen $\left(51^{\circ} 13^{\prime} 16^{\prime \prime}\right.$ N, $4^{\circ} 23^{\prime} 60^{\prime \prime} \mathrm{E}$ ). Coll and subm 1982 by M Van Strydonck. Sample was taken from column of bricks embedded in sandstone at $6 \mathrm{~m}$ above street level. Column is loc in nave of church, built between 530 and 515 BP.

IRPA-496. Vrasene 1

$1600 \pm 60$

Very powdery mortar from "Heilige-Kruis" church, Vrasene, O Vlaanderen $\left(51^{\circ} 13^{\prime} \mathrm{N}, 4^{\circ} 12^{\prime} \mathrm{E}\right)$. Coll and subm 1982 by M Van Strydonck. Sample was taken from Romanesque bench surrounding pillar built between 800 and 767 вP.

IRPA-497. Vrasene 2

$870 \pm 50$

Mortar from same church. Coll and subm 1982 by M Van Strydonck. Sample was taken from chalk blending used during construction period (ca AD 1350) at street level.

General Comment: IRPA-490 and -497 agree with historic age. IRPA-296 is too old but contamination by running water containing carbonate is possible because of location of sample. For IRPA-496, method of "hypothetical fraction" does not work probably because sample is very powdery. If we assume that at first approximation, measured $\delta^{13} \mathrm{C}$ is proportional to abundance of "live" carbonate, we can calculate theoretical date which corroborated historic age, $980 \pm 80$ BP, (Van Strydonck, Dupas, \& DauchotDehon, in press).

\section{REFERENCES}

Baeteman, C, ms, 1981, De Holocene ontwikkeling van de Weselijke kustvlakte (België): PhD dissert, Univ Brussels, 297 p.

Baeteman, C, Verbruggen, C, with Dauchot-Dehon, M, Heylen, J, and Van Strydonck, M, 1979, New approach to the evolution of the so-called surface peat in the Western Coastal Plain of Belgium: Service Geol Belgium. Prof paper, v 11, no. 167, p $2-16$.

Dauchot-Dehon, M, Van Strydonck, M, and Heylen, J, 1981, Institut royal du Patrimoine artistique radiocarbon dates IX: Radiocarbon, v 23, p 345-346.

Klein, J, Lerman, J C, Damon, P E, and Ralph, E K, 1982, Calibration of radiocarbon dates: tables based on the consensus data of the Workshop on Calibrating the Radiocarbon Time Scale: Radiocarbon, v 24, p 103-150.

Folk, R L and Valastro, S, 1976, Successful technique for dating of lime mortar by carbon-14: Jour Field Archaeol, v 3, p 203-208.

Van Strydonck, M, Dupas, M, and Dauchot-Dehon, $\mathbf{M}$, in press, A further step in the radiocarbon dating of old mortars: Inst Royal Patrimoine artistique Bull, in press. 